Z Rheumatol 2016 · 75:859-860

DOI 10.1007/s00393-016-0205-0

Online publiziert: 28. September 2016

๑) Springer-Verlag Berlin Heidelberg 2016

CrossMark

\title{
Elisabeth Märker-Hermann
}

Klinik Innere Medizin IV (Rheumatologie, klinische Immunologie und Nephrologie), HELIOS Dr. Horst Schmidt Kliniken Wiesbaden, Wiesbaden, Deutschland

\section{Infektinduzierte rheumatische Erkrankungen}

Falle positiver Antikörperbefunde für Borrelien oder Yersinien durchgeführt werden. Für die reaktive Arthritis und die Lyme-Arthritis haben in dieser Zeitschrift bereits vor mehr als 10 Jahren Haibel et al. [1] einen in der Praxis sehr gut einzusetzenden diagnostischen Algorithmus publiziert. Die Kollegen haben auch in diesem Zusammenhang wieder betont, dass zur Diagnose einer reaktiven Arthritis weder ein Labortest noch die Kenntnis der Sensitivität und Spezifität des verwendeten Testsystems ausreicht. Es ist immer eine Kombination aus verschiedenen Parametern - und hier v. a. das klinische Erscheinungsbild - erforderlich, das von einem typischen Gelenkmanifestationsmuster und häufig auch extraartikulären Symptomen geprägt ist. Schließlich ist zur Beurteilung der Wertigkeit eines Tests auch die Kenntnis der angenommenen Häufigkeit der Krankheit in der jeweiligen Region (Prätestwahrscheinlichkeit) erforderlich.

Man weiß aus epidemiologischen Studien, dass eine virale Ätiologie für ca. $1 \%$ aller akuten Arthritiden weltweit verantwortlich zu machen ist. Dabei zeigte sich in den vergangenen 10 Jahren eine Veränderung der Inzidenz bestimmter arthritogener Virusinfektionen, wie z. B. eine Abnahme der Rötelnarthritis infolge der Rötelnimpfprogramme zugunsten „neuer“, durch Stechmücken übertragener Alphaviren, die als Folge des weltweiten Tourismus und eines epidemiologischen Shifts gehäuft diagnostiziert werden. Somit ist bei Patienten mit akuter Arthritis eine Reiseanamnese ebenso wichtig wie die Identifikation von potenziellen Risiken, mit Kinderkrankheiten wie Parvovirus B19 oder Hepatitisviren und humanem Immundefizienzvirus (HIV) in
Berührung zu kommen. Eine routinemäßige, ungezielte serologische Antikörperdiagnostik bei Patienten mit einer arthritischen Symptomdauer von weniger als 6 Monaten kann jedenfalls nicht empfohlen werden. So zeigte sich 2011 in einem französischen Studienkollektiv von 806 Früharthritispatienten, bei denen serologisch auf Parvovirus B19, HepatitisB-Virus (HBV) und Hepatitis-C-Virus (HCV) sowie HIV getestet wurde, dass in mehr als $70 \%$ ein Durchseuchungstiter für Parvovirus B19 gefunden wurde. Eine virale Genese der Arthritis wurde hingegen nur bei 2 Patientinnen mit akuter Parvovirus-B19-, bei 3 Patienten mit HBV- und bei 5 Patienten mit HCVInfektion gefunden, wobei die Patienten mit Hepatitisinfektion auch durch erhöhte Transaminasen auffielen [2].

Wie immer in der Medizin gilt auch für die Diagnostik der erregerinduzierten Arthritiden, dass eine gute Anamnese und das klinische Erscheinungsbild die Grundlage für die Beauftragung der nachfolgenden spezifischen Labortests sein sollten.

Es grüßt Sie herzlich

Ihre

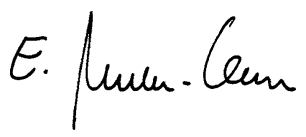

Prof. Dr. Elisabeth Märker-Hermann, Wiesbaden 


\section{Korrespondenzadresse}

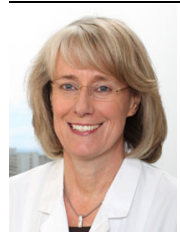

Prof. Dr. med. E. Märker-

Hermann

Klinik Innere Medizin IV (Rheumatologie, klinische Immunologie und Nephrologie), HELIOS Dr. Horst Schmidt Kliniken Wiesbaden Ludwig Erhard Str. 100, 65199 Wiesbaden,

Deutschland

Elisabeth.MaerkerHermann@Helios-Kliniken.de

Interessenkonflikt. E. Märker-Hermann gibt an, dass kein Interessenkonflikt besteht.

\section{Literatur}

1. Haibel H, Rudwaleit M, Sieper J, Zeidler H, Kuipers JG (2004) Diagnostik der reaktiven Arthritis. Z Rheumatol 63:211-215

2. Varache S, Narbonne V, Jousse-Joulin S, Guennoc $X$, Dougados M, Daurès JP, Devauchelle-Pensec V, Saraux A (2011) Is routine viral screening useful in patients with recent-onset polyarthritis of a duration of at least 6 weeks? Results from a nationwide longitudinal prospective cohort study. Arthritis Care Res (Hoboken) 63:1565-1570

\section{K.C. Chung (Ed.) \\ Clinical Management of the Rheumatoid Hand, Wrist, and Elbow}

\section{Springer International Publishing}

2016, 1. Auflage, 330 S., 260 Abb., (ISBN: 978-3-319-26658-9), Hardcover 160,49 EUR, eBook (978-3-319-266602) 118,99 EUR

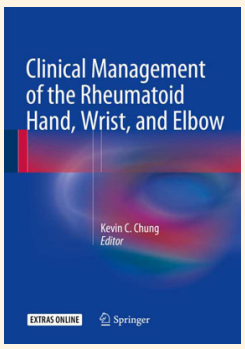

Die Erstausgabe

"Clinical Management of the Rheumatoid Hand, Wrist and Elbow" ist ein über 300-seitiges Kompendium zur operativen Therapie der Hand, des

Handgelenkes und des Ellenbogens bei rheumatoider Arthritis. Das Buch ist in vier große Bereiche gegliedert (Hintergrundwissen, rheumatisches Handgelenk, rheumatische Hand, rheumatischer Ellenbogen). Im ersten Kapitel werden Hintergründe zur rheumatoiden Arthritis dargestellt. Dies inkludiert die Mechanismen der neuesten Medikamente, mögliche Pathomechanismen der rheumatoiden Arthritis und wichtige Felder wie z.B. die prä- und postoperative Medikamententherapie beim chirurgischen Eingriff. Ein eigenes Kapitel für die Indikation und den Zeitpunkt des chirurgischen Eingriffs beim Patienten mit rheumatoider Arthritis wird ebenso suffizient dargestellt wie verschiedene Untersuchungsscores. Der zweite Bereich befasst sich mit dem rheumatischen Handgelenk. Hier werden zunächst verschiedene biomechanische Erklärungsmodelle und Konzepte zum rheumatischen Handgelenk aufgeführt. Danach erfolgt eine detaillierte Beschreibung der verschiedenen Handgelenksregionen inklusive des distalen Radioulnargelenkes. Der Bereich des rheumatischen Handgelenkes wird abgerundet mit einer hervorragenden Falldiskussion, bei der verschiedene Patienten kurz und prägnant dargestellt und dann, nach Abarbeiten der Diagnostik, die entsprechenden Therapieoptionen aufgeführt werden. Hierdurch ist eine Wissensüberprüfung des Lesers möglich. Im dritten Teil des Buches wird die rheumatoide Handdeformität dargestellt. Hier ebenfalls Beginn mit biomechanischen Überlegun- gen zur Entstehung der Deformitäten und Indikationsstellung. In Folge werden neben den Gelenkersatzmöglichkeiten auch die wichtigen Sehneneingriffe vorgestellt. Auch dieser Bereich wird wiederum durch eine fallbasierte Darstellung von verschiedenen Patienten abgerundet. Als letzter Bereich wird, etwas kürzer als die vorhergehenden Anteile, der rheumatische Ellenbogen und die Therapiemöglichkeiten dargestellt.

Die Indikation zu hand- bzw. ellenbogenchirurgischen Eingriffen bei Patienten mit rheumatoider Arthritis ist rückläufig. Nichtsdestotrotz können weiterhin viele Patienten von der operativen Therapie profitieren. Hierzu stellt dieses Buch einen hervorragenden Leitfaden dar. Aufgrund der sorgfältig ausgearbeiteten Kapitel, der sinnvollen Gliederung und der ansprechenden Abbildungen kann dieses Buch jedem Chirurgen, der eine operative Therapie bei rheumatoiden Patienten durchführt, nur wärmstens empfohlen werden. Auch internistische Rheumatologen können aufgrund der guten Darstellung der operativen Techniken von diesem Buch durchaus profitieren.

F. Unglaub (Bad Rappenau) 